\title{
Head striking during fish capture attempts by Steller sea lions and the potential for using head surge acceleration to predict feeding behavior
}

\author{
John P. Skinner ${ }^{1, *}$, Sarah E. Norberg ${ }^{1}$, Russel D. Andrews ${ }^{1,2}$ \\ ${ }^{1}$ Alaska SeaLife Center, PO Box 1329, Seward, Alaska 99664, USA \\ ${ }^{2}$ School of Fisheries and Ocean Sciences, University of Alaska, Fairbanks, Alaska 99775-7220, USA
}

\begin{abstract}
After declining by over $80 \%$ in the last 30 yr, Steller sea lion Eumetopias jubatus (SSL) populations in Alaska remain low with no sign of recovery. To better examine the potential effects of nutritional stress, foraging studies are needed. Currently available biotelemetry techniques are either incapable of precisely quantifying prey captures or have not been validated for SSLs. Our objectives were to (1) determine whether SSL fish-capture attempts (FCAs) produce a head surge acceleration signal; $(2)$ determine whether acceleration of the head relative to the body (differential) produces a more distinct signal than head acceleration alone (dynamic); and (3) develop a method for predicting FCAs using accelerometry. We attached accelerometers to the head and torso of 2 captive SSLs at the Alaska SeaLife Center and allowed them to feed on live fish. For the first SSL, we used video to classify FCAs during foraging trials and modeled the association between FCAs and parameters created using both dynamic and differential acceleration. Model coefficients were used to predict FCAs for a second SSL, and the results were compared with video. Head acceleration spikes were found in all FCAs. The dynamic model performed slightly better than the differential model at correctly predicting FCAs. Although the model did not perform exceptionally at identifying individual FCAs, it did predict at least 1 FCA in $96 \%$ of fish-chasing events and made only 1 false FCA prediction during $392 \mathrm{~min}$ of non-foraging. This study demonstrates that head accelerometry is useful for estimating SSL foraging effort.
\end{abstract}

KEY WORDS: Foraging behavior · Remote monitoring $\cdot$ Eumetopias jubatus · Acceleration data logger $\cdot$ Signal detection

\section{INTRODUCTION}

Knowing when, where, and how a species acquires food is fundamental to understanding its ecology and is critical to its conservation. Steller sea lion Eumetopias jubatus (SSL) populations in Alaska, USA, experienced a dramatic decline starting in the mid-1970s. In 1997, the western stock was listed as endangered under the US Marine Mammal Protection Act (Loughlin 1998). Although the precipitous decline has abated, there has been no sign of recovery (Fritz et al. 2008), and it has been proposed that nutritional stress due to changes in the prey community may be currently affecting the population dynamics of SSLs (Holmes et al. 2007,
Atkinson et al. 2008). To examine this hypothesis, information about SSL prey capture effort and success is needed. As with most studies of marine species, these questions are best answered using biotelemetry techniques (Cooke et al. 2004, Naito 2004).

In part because of their simplicity, time-depth recorders have become widely used for foraging studies of marine species (Kooyman 2004). Information about the depth (e.g. Croxall et al. 1988, Wilson et al. 1993) and profile shape (Schreer \& Testa 1995, Schreer et al. 2001) of dives can provide some insights into a species' foraging strategies (e.g. benthic versus pelagic) and allows an estimation of time spent foraging versus commuting. These methods only provide a broad estimate of forag- 
ing effort and no information about prey capture success. To understand the economy of an animal's foraging strategy, methods that offer a more precise estimate of the timing of prey captures are needed.

Abrupt changes in depth (i.e. wiggles) have been used to denote prey capture attempts for king penguins Aptenodytes patagonicus and Adélie penguins Pygoscelis adeliae (Bost et al. 2007), northern elephant seals Mirounga angustirostris (Horsburgh et al. 2008), and humpback whales Megaptera novaeangliae (Goldbogen et al. 2008). This method may be less useful, however, if an animal does not give chase while feeding, such as when hunting by ambush or in a large, dense prey field. Measurement of stomach temperature (Wilson et al. 1992) allows detection of prey ingestion in pelagic sea birds (Weimerskirch et al. 1994) but may be less useful for pinnipeds because of reduced or absent temperature drops during 2 or more sequential feedings and difficulties with maintaining the stomach temperature transmitter in the animal's stomach (Andrews 1998, Kuhn \& Costa 2006). Promising results have come from measurement of jaw position using a mandibular sensor (Wilson et al. 2002), and this method has been used to estimate successful prey ingestions for captive and free-ranging pinnipeds (Liebsch et al. 2007). However, a proper validation of this method may still be required to test its reliability for predicting ingestions in free-ranging animals. Naito (2007) recently summarized methods previously used for foraging studies of marine mammals and birds and suggested that further developments are necessary.

Accelerometry has quickly become a popular tool for measuring animal movement and orientation as a means to infer behavior (e.g. Yoda et al. 2001). Data from acceleration data loggers have been used to examine repetitive behaviors such as wing beat strokes (Ropert-Coudert et al. 2004) or flipper strokes (Davis et al. 2001, Williams et al. 2004). Less stereotypical behaviors, such as head movements during feeding, have also been examined. Suzuki et al. (2009) measured acceleration of the head and lower mandible of captive hooded seals Cystophora cristata during underwater fish feeds. Maxima in mandible and head acceleration were found to provide the precise timing of feeding events. However, this method was not robustly tested, and an objective method for analyzing large data sets collected from animals foraging on live fish was not provided.

During research on captive animals at the Alaska SeaLife Center, we have observed that SSLs use a quick extension of the head (i.e. head-striking) to grab live fish prey. Analysis of video footage confirmed that in many cases the head accelerated at a greater rate or independently of the overall movement of the body.
This exaggerated head movement appeared to be limited to times of prey capture and therefore could provide a distinct signal for identification of fish-capture attempts (FCAs). With an interest in developing a new tool for predicting prey capture effort in wild SSLs, our objectives in this study were to (1) determine whether head-striking during FCAs produces a discernable head surge acceleration signal; $(2)$ determine whether surging acceleration signals of the head relative to the body during prey capture are more distinct than signals produced by the head alone; and (3) develop a simple, automated method for predicting when FCAs occur using surge acceleration signatures.

\section{MATERIALS AND METHODS}

Foraging trials. Between March and May of 2007 and 2008, live fish were fed to 2 adult female captive SSLs equipped with acceleration data loggers at the Alaska SeaLife Center in Seward, Alaska, USA. The masses for these female SSLs, named Kiska and Sugar, ranged from 193 to 207 and 218 to $230 \mathrm{~kg}$, respectively, during the 2 periods of conducting trials. SSL surging acceleration data were collected using customized Mk10 data loggers (Wildlife Computers) containing a tri-axial accelerometer chip (Freescale). We attached 2 acceleration data loggers to each SSL: one fixed dorsally to the torso, posterior to the axilla, and the other placed on the crest of the head (Fig. 1). In 2007, the head accelerometer was connected to the torsomounted data logger via a $3 \mathrm{~mm}$ diameter, $120 \mathrm{~cm}$ long cable, enabling recording of acceleration of both the torso and the head. Depth was recorded to the nearest

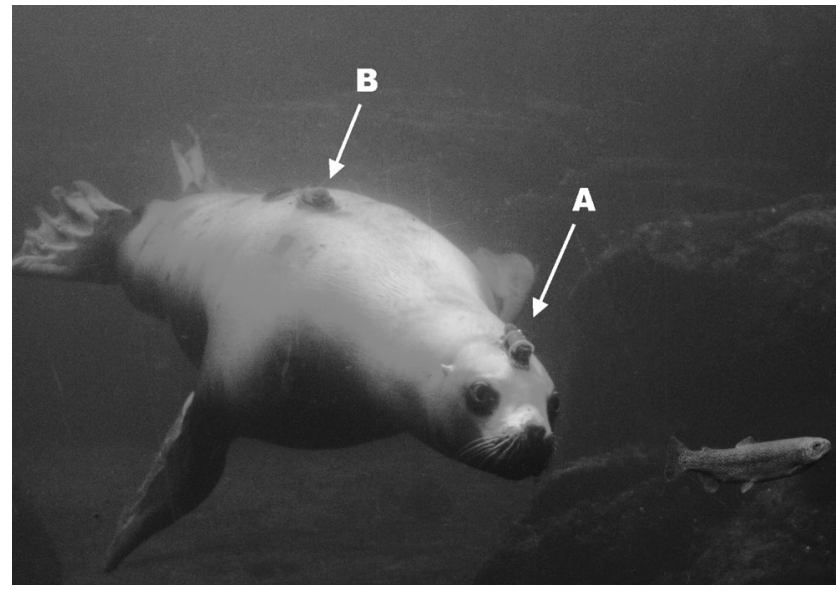

Fig. 1. Eumetopias jubatus. A captive Steller sea lion (Sugar) chasing a $\sim 300 \mathrm{~g}$ rainbow trout on 7 May 2008. Arrows indicate data loggers on (A) the head and (B) the torso. These attachment locations were used during 2007 and 2008, although in 2007 trials, a cable connected a remote head accelerometer sensor to the torso data logger 
$0.5 \mathrm{~m}$ at a frequency of $2 \mathrm{~Hz}$, and head and torso $y$-axis (i.e. surge) acceleration was logged at $32 \mathrm{~Hz}$ with 8 bit resolution over the range of -4 to +4 gravitational units (g). In 2008, 2 separate data loggers were attached in the same locations as in 2007. These instruments recorded depth to the nearest $0.5 \mathrm{~m}$ at $8 \mathrm{~Hz}$ and acceleration at $64 \mathrm{~Hz}$ with 10 bit resolution (range, -4 to $+4 \mathrm{~g}$ ). Instruments were secured to a neoprene basepad which provided a surface for attachment to the fur using 5 min epoxy (ITW Devcon).

For each trial, live coho Oncorhynchus kisutch or Chinook salmon O. tshawytscha, Arctic char Salvelinus alpinus, or rainbow trout O. mykiss weighing on average between 30 and $550 \mathrm{~g}$ were released into the SSL exhibit (a $5 \mathrm{~m}$ deep, $605000 \mathrm{l}$ aquarium) individually or in groups of up to 4 at a time through a $13 \mathrm{~m}$ by $10 \mathrm{~cm}$ diameter PVC tube. Because the sizes of individual fish were not recorded at the time of release, their length was estimated on video by comparing their size relative to the diameter of the PVC tube. Following each fish release, 1 of the SSLs was instructed by its trainer to find and ingest the fish. Foraging behavior was recorded through the exhibit's underwater viewing window using digital video cameras. We defined start of a chase as $1 \mathrm{~s}$ prior to the time when the SSL changed its velocity so that its trajectory intersected that of the fish. Chasing ended $1 \mathrm{~s}$ after the fish had been ingested or if the SSL appeared to no longer chase the fish. During a chase, each instance when the SSL opened its mouth within $5 \mathrm{~cm}$ of a fish was defined as an FCA, irrespective of capture success. The time of each FCA was recorded to the nearest $1 \mathrm{~s}$ upon review of digital video. During foraging trials, times when the SSL was out of view of the camera were excluded from analyses. The time on the video tape was synchronized with the data logger by comparing depth readings to observed diving behavior of SSLs on video. We allowed the SSLs to swim freely when fish were not present to collect additional acceleration data during nonforaging behaviors. No digital video was recorded during these non-foraging periods.

Data handling. Data were processed using Visual Basic for Access (Microsoft) and analyzed with Systat v11 for Windows (SYSTAT Software). Dive depth data were corrected for pressure transducer drift using the minimum depth recorded during a 5 min moving window. A linear calibration was used to convert tag acceleration units to $g$ by using minimal and maximal values attained while aligning the surge axis with the direction of the force of gravity (i.e. -1 to $+1 \mathrm{~g}$ ). A $0 \mathrm{~g}$ correction was attained during each trial by asking the SSL to lie prostrate and motionless on a level surface. In 2008, synchronous mechanical taps on the head and torso data loggers were compared and used to correct time differences between the head and torso data loggers.
The mean acceleration value in a $1 \mathrm{~s}$ moving window centered on each data point was used to estimate the gravitational component of acceleration at that point (Yoda et al. 2001). This value was subtracted from each data point to attain an estimate of dynamic acceleration. To measure head-surging behavior, we evaluated dynamic head surge (dynamic) acceleration and head minus torso dynamic surge (differential) acceleration. For both metrics, rate of change in acceleration (i.e. derivative) was calculated for each acceleration observation by subtracting the previous acceleration value and multiplying the resulting value by the sample frequency.

We used a 2 s moving data window to summarize and analyze SSL surging behavior. For each window, dynamic and differential acceleration were separately evaluated to determine (1) maximum acceleration (i.e. positive surge maximum); (2) minimum acceleration; (3) acceleration range (maximum minus minimum); (4) acceleration mean; (5) maximum derivative; (6) mean of the absolute value of all window derivatives; (7) positive integral area under the acceleration maximum; (8) positive integral in the entire window; (9) maximum surge integral area dominance, which was calculated as the ratio of maximum surge integral area (7) to positive window integral area (8) and; (10) absolute value of time lag between window maximum and minimum. The derivations of these parameters are depicted in Fig. 2.

For data analyses, we excluded all $2 \mathrm{~s}$ data windows that contained data with depths $\leq 1 \mathrm{~m}$. For data collected during foraging trials, if the SSL was not visible during the entire window interval, the window was excluded. Each window was classified as an FCA or a non-FCA, based on whether an FCA was observed on the video during that time window. Additionally, we classified a window as 'during chase' if any portion of the window occurred during an observable fish chase and 'not during chase' if otherwise. During periods of non-foraging, all windows were classified as non-FCA and 'not during chase.'

FCA prediction model. We used data collected on Kiska to develop 2 separate FCA prediction models for dynamic and differential acceleration data. Each data window was assigned a response of ' 1 ' if it was classified as an FCA and a ' 0 ' if the observation did not include an FCA. Because we were interested in training the model using distinct prey capture signals, non-FCA data occurring during a fish chase were excluded from the model. The response was modeled against the window parameters using a general linear model with a logit link (i.e. logistic regression). Parameter interactions were not tested in the model. All independent variables were examined for normality and, if necessary, a natural logarithm was applied to transform them prior to analyses. Suitable parameters were chosen us- 


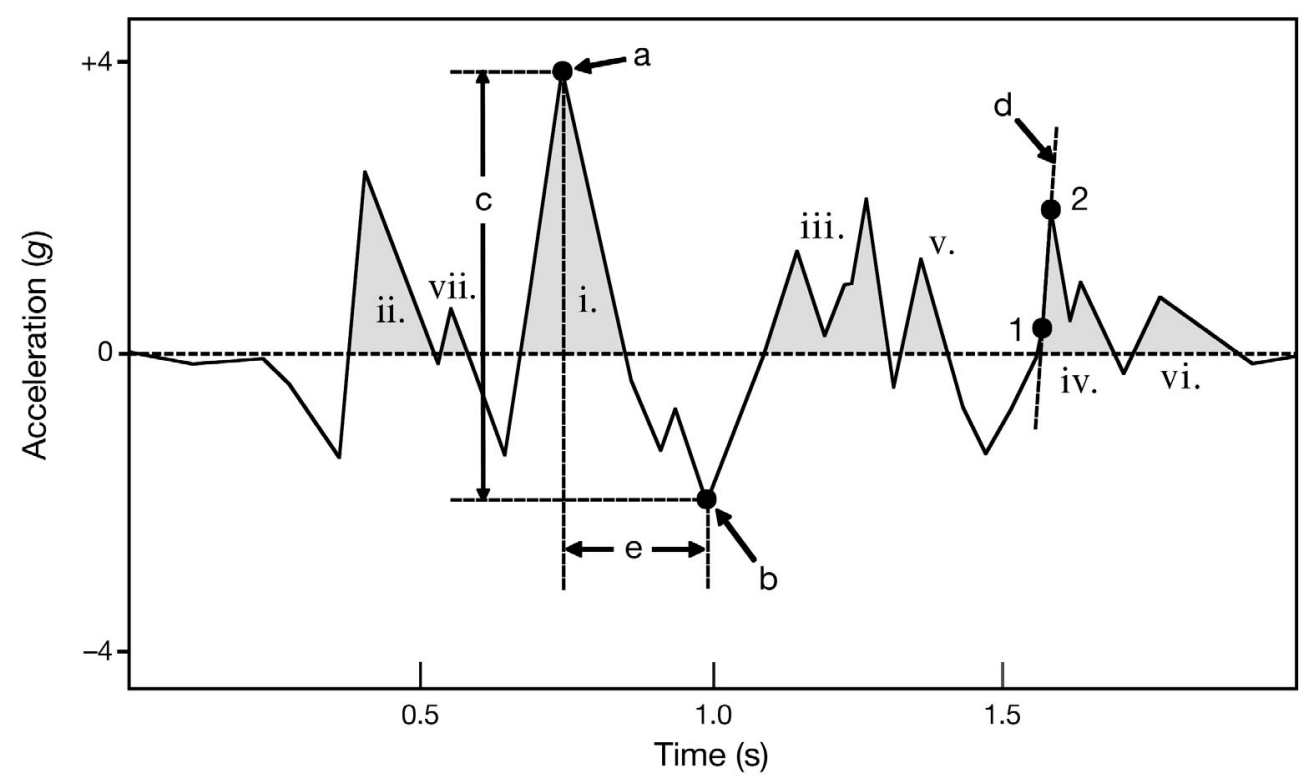

Fig. 2. Eumetopias jubatus. Generalized depiction of dynamic head acceleration in a Steller sea lion during a $2 \mathrm{~s}$ window. The parameters created to describe head acceleration within each window include: (a) maximum acceleration, (b) minimum acceleration, (c) acceleration range, (d) maximum derivative of acceleration (slope of the line between points 1 and 2), (e) time lag between maximum and minimum acceleration. Individual surge maxima were defined as consecutive points $>0 \mathrm{~g}$ (shaded) and are indicated by roman numerals to indicate their ranking based on their maximum acceleration. Window maximum surge integral area is the shaded area of peak (i). Window positive integral area was calculated as the sum of integral area for all peaks (total shaded area of [i] through [vii]). Maximum surge dominance was calculated as the maximum surge integral area (i) divided by window positive integral area

ing backward stepwise model selection $(\alpha=0.01)$.

Data collected with Sugar were used to validate the stepwise chosen model parameters for both the dynamic and differential acceleration models. For each 2 s observation window, model coefficients were used to calculate a predicted FCA probability scaled from 0 to 1 . We classified an observation as a predicted FCA if the predicted probability was $>0.5$ or as a predicted non-FCA otherwise. The performances of dynamic and differential acceleration models were evaluated and compared by tabulating performance statistics using the numbers of actual FCAs that were correctly predicted (true positive), non-FCAs that were correctly predicted (true negative), actual FCAs incorrectly predicted as non-FCAs (false negative), and actual nonFCAs incorrectly predicted as FCAs (false positive) by the model. These statistics included the following expressed as percentages: (1) accuracy, which is the sum of true positives and true negatives divided by the total number of observations; (2) precision, the number of true positives divided by the sum of true and false positives; (3) sensitivity, the number of true positives divided by the sum of true positives and false negatives; (4) specificity, the number of true negatives divided by the sum of true negatives and false positives; and (5) false positive rate, the number of false positives divided by the total number of non-FCAs.

\section{RESULTS}

During foraging trials, Kiska and Sugar were observed on video for 173.7 and $168.2 \mathrm{~min}$, respectively. For 132 fish releases during Kiska's trials (n = 11), only $12.8 \mathrm{~min}$ of chase time occurred at a depth $>1 \mathrm{~m}$ and could be included in the analyses. From the video, we noted 148 separate FCAs by Kiska. Due to some FCAs occurring within the same $2 \mathrm{~s}$ data window or at a shallow depth, there were only 126 distinct FCA windows included for analysis. The non-chasing data windows included in the model $(\mathrm{n}=4495)$ came from times when Kiska was below $1 \mathrm{~m}$ and was observed on video not chasing fish (102.7 $\mathrm{min}$ ) or when the exhibit was known to be free of fish (47.1 min). The model was validated using $9.8 \mathrm{~min}$ of chasing and $391.7 \mathrm{~min}$ of non-chase data from Sugar as she dove below $1 \mathrm{~m}$. Sugar performed 133 FCAs on video resulting in 92 data windows being classified as having at least 1 FCA.

We examined dynamic and differential acceleration data collected during periods when SSLs were chasing fish on video and noted what behavior corresponded to each acceleration spike (Fig. 3). We found that most FCAs were associated with abrupt changes in acceleration with higher maxima and minima values than data associated with other behaviors while diving. 
During an initial fish-strike by the SSL, there was at least 1 large positive and/or negative spike in acceleration. Often, abrupt changes in acceleration continued for some length of time after the initial spike. We found that these signals were associated with the SSL manipulating the fish (e.g. improving grip or turning the fish in the mouth; Fig. 4 intervals labeled 'b'). Following many but not all prey captures, a single or series of distinct positive acceleration spikes occurred at intervals between 0.5 and $0.75 \mathrm{~s}$ associated with head extensions during swallowing (Fig. 4, 'spikes' labeled ' $\mathrm{C}$ '). Overall, dynamic and differential acceleration plotted against time appeared very similar.

A logarithmic transformation was necessary to normalize all parameters except average window acceleration. In the dynamic acceleration model, FCAs were positively associated with window acceleration range (Hotelling's $t$-ratio $=5.22, \mathrm{p}<0.001$ ) and the mean absolute value of window derivatives ( $t$-ratio $=3.97$, $\mathrm{p}<0.001$ ), and the model had a high degree of fit to the data (McFadden's $\rho^{2}=0.74$ ). The resulting dynamic acceleration model had the following form:

$$
\pi=\frac{1}{1+\mathrm{e}^{-\left[-4.810+\left(2.049 \times X_{1}\right)+\left(1.487 \times X_{2}\right)\right]}}
$$

where $\pi=$ probability that the observation is associated with an FCA (1 = FCA; $0=$ not chasing fish), $X_{1}=$ logtransformed window acceleration range, and $X_{2}=\log$ transformed window mean of derivative absolute values.

Using differential acceleration data to develop a separate model, FCAs were positively associated with window acceleration range $(t$-ratio $=15.36, \mathrm{p}<0.001)$ while being negatively correlated with maximum surge integral area dominance $(t$-ratio $=-3.11, \mathrm{p}=$ 0.002). This model also had a high degree of fit (McFadden's $\rho^{2}=0.74$ ) with the following coefficients:

$$
\pi=\frac{1}{1+\mathrm{e}^{-\left[-2.507+\left(3.426 \times X_{1}\right)+\left(-2.696 \times X_{2}\right)\right]}}
$$

where $\pi$ and $X_{1}$ are as in Eq. (1), and $X_{2}=\log$ transformed maximum surge integral area dominance.

With Sugar's data, an FCA prediction probability was calculated for each data window $(n=12045)$ using the logistic regression model coefficients. For each model, predicted FCA classifications of each $2 \mathrm{~s}$ observation window were compared to their actual classification, either FCA or non-FCA (Table 1). The dynamic model had high accuracy (99.1\%) and specificity $(99.3 \%)$ but had some difficulty in resolving known
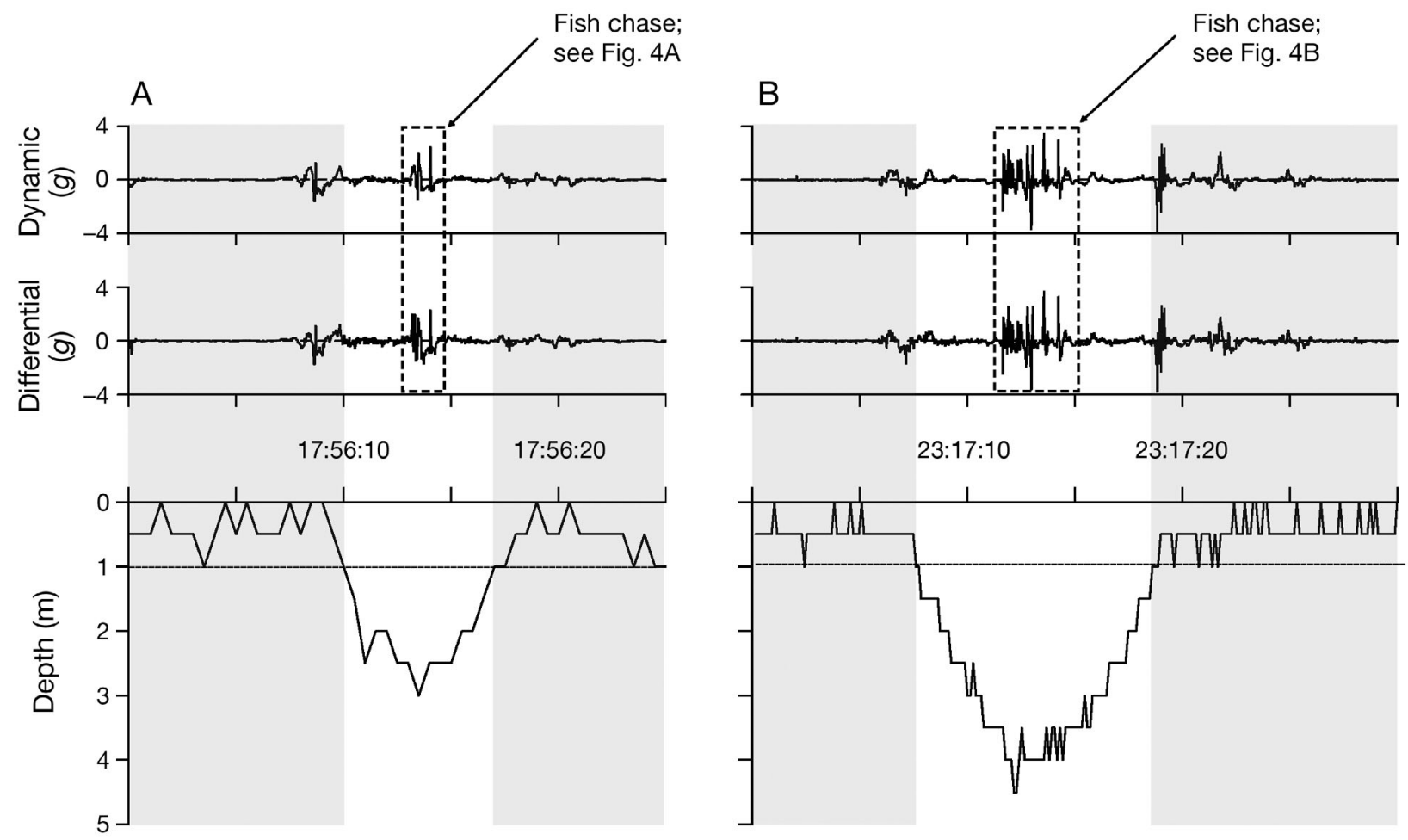

Fig. 3. Eumetopias jubatus. Steller sea lion dynamic head acceleration (dynamic, top), differential head-torso acceleration (differential, center), and depth (bottom) over time during two different live fish feeding trials (A and B). Data occurring at depths $\leq 1 \mathrm{~m}$ were excluded from analyses (shaded). Time intervals when the SSL was chasing a fish are shown by a dotted box, and expanded views are provided in Fig. 4 

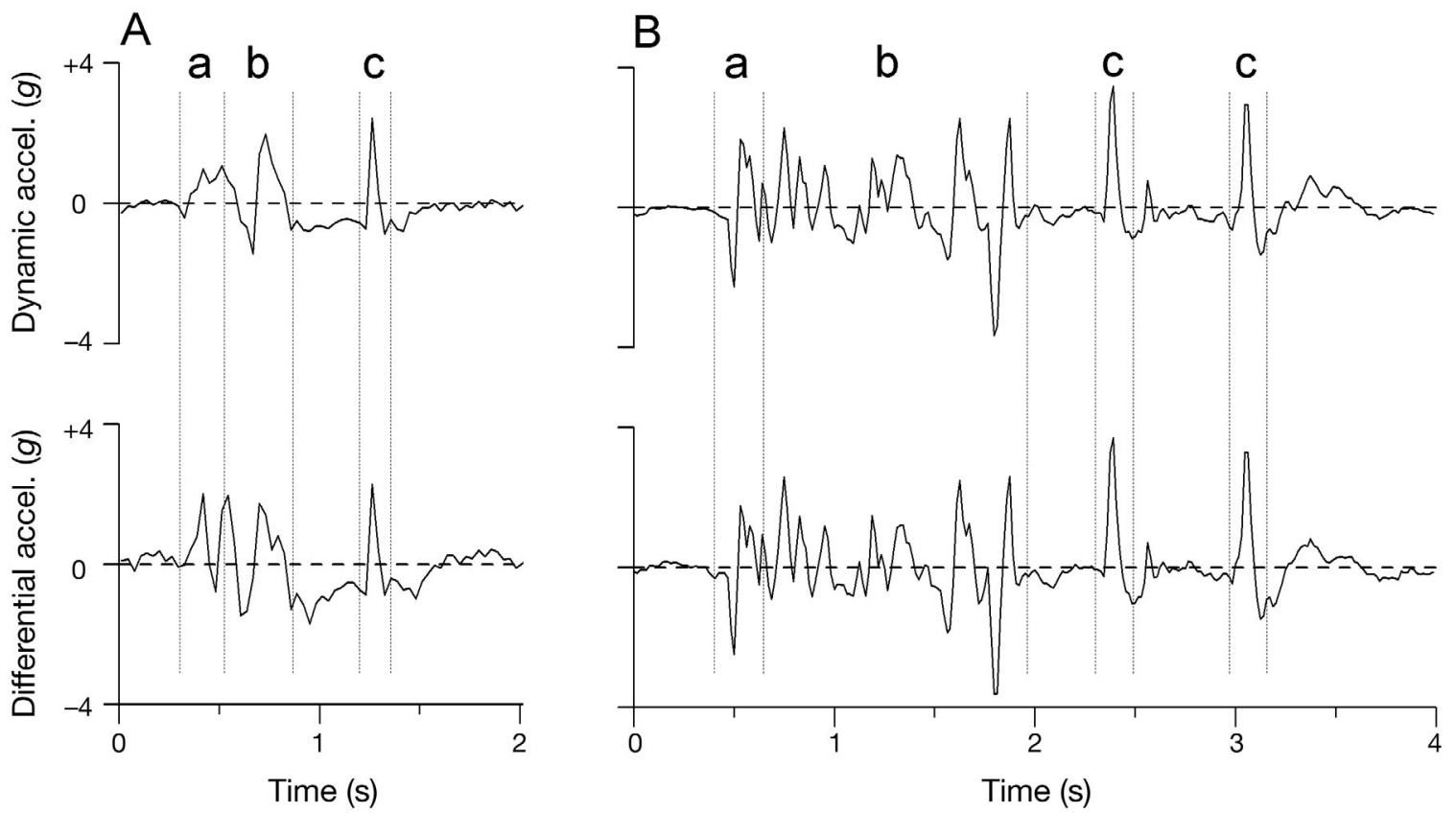

Fig. 4. Eumetopias jubatus. Dynamic head (top) and differential head-torso (bottom) acceleration over 2 separate time intervals during which the Steller sea lion was chasing a fish. During interval (A), the SSL captured a 175 g Chinook salmon, and during (B), she captured a $350 \mathrm{~g}$ rainbow trout. Observed behaviors associated with these signals are: (a) successful prey capture, (b) head movements during repositioning of fish in the mouth, and (c) visible head extension during swallowing

FCAs (sensitivity $=81.5 \%$ ). Additionally, the dynamic model incorrectly predicted 86 non-FCAs to be FCAs (false positive rate $=0.7 \%$, precision $=46.6 \%$ ); however, 85 of these false positives occurred during a fish chase. Similar to the dynamic model, the differential model was very accurate $(99.1 \%)$ and had a high specificity $(99.2 \%)$ but was slightly less sensitive $(79.3 \%)$. However, the differential model had a slightly greater tendency to over-predict FCAs, as it incorrectly classified 95 observations as FCAs (false positive rate $=$ $0.8 \%$, precision $=43.5 \%$ ). Of these 95 false positives, 86 occurred during periods of fish chasing.

\section{DISCUSSION}

We found a positive spike in both dynamic and differential acceleration during each SSL FCA, although the magnitude of these spikes varied considerably across different fish strikes. This confirmed our speculations, originally based on observations of captive SSL foraging, that they strike out with their heads during FCAs. We were surprised, however, to find that FCAs often produced a series of large-amplitude spikes rather than a single, dominant spike. These surge spike groups appeared to be the combination of an initial FCA and the subsequent fish handling in the mouth. The duration of this manipulation signal appeared to increase in duration with the size of the fish, although we did not test for this effect. Examples of FCAs for fish of different sizes are illustrated in Fig. 4, with a $175 \mathrm{~g}$ fish handled in panel A and a $350 \mathrm{~g}$

Table 1. Eumetopias jubatus. Confusion matrices. The parameters from the differential and dynamic acceleration models built on the data of a Steller sea lion (Kiska) were used to produce fish-capture attempt (FCA) predictions for every $2 \mathrm{~s}$ data window from a second sea lion (Sugar; $\mathrm{n}=12045)$. Model predictions were compared to the actual window classifications as verified by the video to evaluate model performances. Dynamic and differential acceleration models appeared to perform similarly

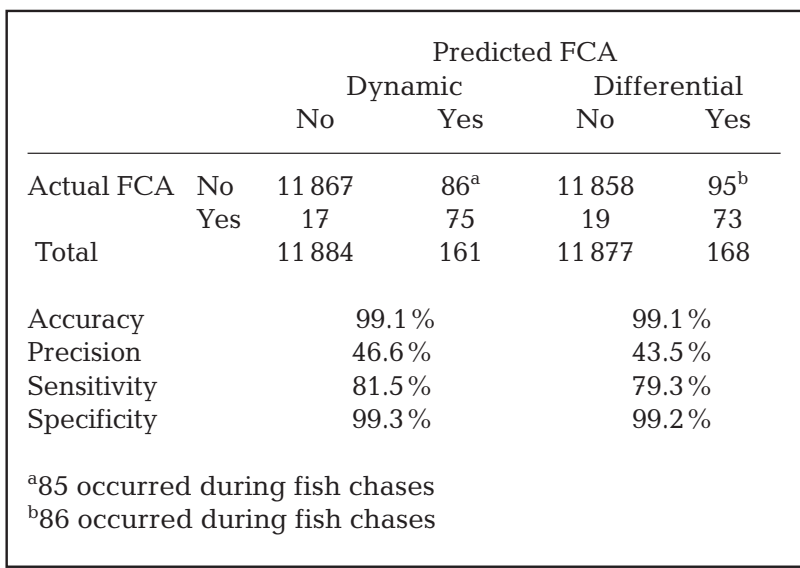


fish handled in panel B. Distinct positive spikes in acceleration also occurred following the initial FCA spikes as a result of the SSL extending its neck to swallow the fish. Although we did not address this in the current study, the combination of these signals may provide indications of successful prey capture.

The parameters selected to describe FCAs in both dynamic and differential models were consistent with what we found when inspecting FCA acceleration signals. In surge acceleration plots, FCAs appeared as a series of large-amplitude swings in acceleration from positive to negative (Fig. 3), which accounts for a strong positive association between FCAs and acceleration range in both models. In the case of the dynamic model, there was a positive association of FCAs with the absolute rate of change in acceleration. This parameter best described the quick oscillations in acceleration between positive and negative values. For the differential model, FCAs were distinguished by being negatively associated with large dominant positive acceleration integral area. Again, this is likely because FCAs created several sequential peaks of similar size.

Our initial foraging observations led us to believe that SSL head-torso differential acceleration might be a better predictor of FCAs than measurements at the head alone. This is because we hypothesized that surging of the head and body during flipper strokes might produce signals similar to surging of the head during FCAs. However, the differential model offered no advantage over the dynamic model in terms of identifying signals that predict FCAs. In fact, validation performance metrics (e.g. accuracy, precision) suggest that the dynamic model performed as well, if not slightly better than, the differential model. This is likely because acceleration of the head during an FCA is far greater in amplitude and much shorter in duration than the acceleration signals produced during normal swimming behaviors. Furthermore, increased propulsion of the body by flipper strokes during an FCA may accentuate the dynamic acceleration signal, which could explain why the dynamic model performed slightly better.

In this study we used a very strict definition for an FCA in order to be objective and conservative when classifying the SSL intent to capture a fish. Consequently, some events during fish chases that may have looked like head-striking at prey but that we did not classify as FCAs because the SSL did not open its mouth were not distinguishable from FCAs by the model, and therefore the model had a relatively high false positive rate. However, we reemphasize that of the 86 false positives from the dynamic model used on Sugar's data, only one occurred outside of times when the SSL was chasing fish. The model therefore appears to be extremely precise and accurate in predicting fish-chasing versus non-fish-chasing behavior.
There were 92 actual FCA windows for Sugar; however, the dynamic model only predicted FCAs in 75 of these windows (sensitivity $=81.5 \%$ ). Despite this tendency to overlook some FCAs, the model performed well at predicting prey capture on a by-fish-chase basis. Of 76 fish chases during which we observed at least 1 FCA by Sugar, the dynamic model predicted 1 or more FCAs in 73 of these chases (96\% prediction success). This is attributed to the occurrence of several FCAs during each chase and the tendency of the model to predict FCAs based on behaviors during foraging that we did not identify as FCAs.

We acknowledge the limitations of using captive animals when developing a method for measuring behavior of their free-ranging counterparts. In this study, we noted several behaviors that would not likely occur outside of a captive study. First, our SSLs became less motivated near the end of feeding trials. This is likely a result of SSLs being fed near or past the point of satiation during trials. Second, there were cases when the SSL appeared to play with fish rather than immediately eat them. Although FCAs did occur between these periods of play, other behaviors, such as nosing and intentional releasing of the fish produced signals that we consider to be less relevant for the study of wild sea lions. Similar play behaviors with non-prey objects may have occurred during periods of nonforaging, and although the dynamic model did not predict FCAs during these periods, the differential model did make 3 false positive predictions during non-foraging times. Without video, we were unable to evaluate behaviors that resulted from interacting with visitors or playing with toys left in the exhibit for enrichment. Finally, some fish that we released during foraging trials appeared to be disoriented when leaving the PVC tube. In some cases, they even remained motionless upon being approached by the SSL. We did note, however, that even when fish failed to make the typical attempt to escape, the SSL still made a clear head strike when attempting to grab the fish. Although these problems are clearly limitations caused by working with captive animals, their most likely effect was to reduce, not improve, the performance of our predictive model. Therefore, we suggest that the method may work even better at predicting the foraging behaviors of free-ranging SSLs.

The effect of acceleration data sampling rate on the ability to identify a particular behavior has not yet been thoroughly examined. As was found with measurements of dive behavior (Boyd 1993), the appropriate rate to sample acceleration is certain to depend on the question being addressed and the duration of the behavior being measured. When relying on acceleration data to capture quick movements within split seconds, an inadequate sampling rate could result in an 
underestimation of the magnitude of the acceleration produced by the movement. The adequate performances of the models in this study suggested that a $32 \mathrm{~Hz}$ sampling rate was sufficient to measure headsurging behavior in SSLs. However, we tested the effect of sampling frequency (32 versus $64 \mathrm{~Hz}$ ) on the mean magnitudes for parameters used in the dynamic acceleration model. Using all FCAs for both SSLs, we found that the log-transformed average absolute change in acceleration was significantly lower at $32 \mathrm{~Hz}$ (pooled $t$-test, $t=2.29, \mathrm{p}=0.02$ ). Log-transformed acceleration range was also slightly lower at $32 \mathrm{~Hz}$ $($ mean $=1.23)$ compared to the mean at $64 \mathrm{~Hz}($ mean $=$ $1.33, t=0.90, \mathrm{p}=0.36$ ). A more careful examination of the effects of sampling rate on the ability to measure FCAs is needed, given that there is a trade-off between sampling rate and the duration of time over which data can be collected.

In conclusion, this study has shown that SSLs do strike at fish with a forward surge of the head during FCAs and that accelerometry can be used to measure this behavior. Furthermore, head acceleration signals associated with foraging were sufficiently distinguishable from other SSL behaviors to allow successful prediction of FCAs. We found that measurement of acceleration at the torso, used to estimate head-torso differential acceleration, is not necessary for making accurate FCA predictions. In addition to measuring the surging acceleration of the head, we suggest that including swaying and heaving head acceleration may further improve this method. On occasion, SSLs were observed sweeping the head, rather than striking, during fish captures, suggesting that the greatest acceleration signal produced during an FCA may not always be in alignment with the head surge axis. Although an examination of model performance using free-ranging SSLs and a wider range of prey types is needed, the method we present here shows much potential for estimating FCAs in SSLs and may serve as a useful tool for examining nutritional limitations in the western SSL population.

Acknowledgements. Funding for this study came from grants provided to the Alaska SeaLife Center (ASLC) by NOAA-NMFS. We thank the staff at the ASLC for their continued support, especially the efforts of B. Bernhardt, N. Charbonneau, C. Cherry, E. DeCastro, H. Down, H. Ferren, C. Goertz, L. Hartman, R. Harris, R. Hocking, J. Jenniges, B. Long, E. Moundalexis, B. Mullaly, M. Peterson, C. Phillips, L. Smith, A. Stephens, D. Trobaugh, P. Tuomi, and many interns and volunteers. We are grateful to MariCal and Fort Richardson Hatchery for generously providing live fish. This research was conducted under NOAA Fisheries Office of Protected Resources permit no. 881-1745 and Alaska Department of Fish and Game permit no. CF-08037, and was approved by the ASLC Institutional Animal Care and Use Committee.

\section{LITERATURE CITED}

Andrews RD (1998) Remotely releasable instruments for monitoring the foraging behavior of pinnipeds. Mar Ecol Prog Ser 175:289-294

Atkinson S, DeMaster DP, Calkins DG (2008) Anthropogenic causes of the western Steller sea lion Eumetopias jubatus population decline and their threat to recovery. Mammal Rev 38:1-18

Bost CA, Handrich Y, Butler PJ, Fahlman A, Halsey LG, Woakes AJ, Ropert-Coudert Y (2007) Changes in dive profiles as an indicator of feeding success in king and Adélie penguins. Deep-Sea Res II 54: 248-255

$>$ Boyd IL (1993) Selecting sampling frequency for measuring diving behavior. Mar Mamm Sci 9:424-430

$>$ Cooke SJ, Hinch SG, Wikelski M, Andrews RD, Kuchel LJ, Wolcott TG, Butler PJ (2004) Biotelemetry: a mechanistic approach to ecology. Trends Ecol Evol 19:334-343

Croxall JP, Davis RW, O'Connell MJ (1988) Diving patterns in relation to diet of gentoo and macaroni penguins at South Georgia. Condor 90:157-167

> Davis RW, Fuiman LA, Williams TM, Le Boeuf BJ (2001) Three-dimensional movements and swimming activity of a northern elephant seal. Comp Biochem Physiol A Mol Integr Physiol 129:759-770

Fritz L, Lynn M, Kunisch E, Sweeney K (2008) Aerial, ship, and land-based surveys of Steller sea lions (Eumetopias jubatus) in Alaska, June and July 2005-2007. US Dept Commerce, NOAA Tech Memo, NMFS-AFSC-183

Goldbogen JA, Calambokidis J, Croll DA, Harvey JT and others (2008) Foraging behavior of humpback whales: kinematic and respiratory patterns suggest a high cost for a lunge. J Exp Biol 211:3712-3719

> Holmes EE, Fritz LW, York AE, Sweeney K (2007) Age-structured modeling reveals long-term declines in the natality of western Steller sea lions. Ecol Appl 17:2214-2232

> Horsburgh JM, Morrice M, Lea Ma, Hindell MA (2008) Determining feeding events and prey encounter rates in a southern elephant seal: a method using swim speed and stomach temperature. Mar Mamm Sci 24:207-217

Kooyman CA (2004) Genesis and evolution of bio-logging devices: 1963-2002. Mem Natl Inst Polar Res 58 (Spec Iss):15-22

Kuhn CE, Costa DP (2006) Identifying and quantifying prey consumption using stomach temperature change in pinnipeds. J Exp Biol 209:4524-4532

Liebsch N, Wilson RP, Bornemann H, Adelung D, Plötz J (2007) Mouthing off about fish capture: jaw movement in pinnipeds reveals the real secrets of ingestion. Deep-Sea Res II 54:256-269

Loughlin TR (1998) The Steller sea lion: a declining species. Biosphere Conserv 1:91-98

Naito Y (2004) New steps in bio-logging science. Mem Natl Inst Polar Res 58 (Spec Iss):50-57

> Naito Y (2007) How can we observe the underwater feeding behavior of endotherms? Polar Sci 1:101-111

Ropert-Coudert Y, Grémillet D, Kato A, Ryan PG, Naito Y, Le Maho Y (2004) A fine-scale time budget of Cape gannets provides insights into the foraging strategies of coastal seabirds. Anim Behav 67:985-992

Schreer JF, Testa JW (1995) Statistical classification of diving behavior. Mar Mamm Sci 11:85-93

Schreer JF, Kovacs KM, O'Hara Hines RJ (2001) Comparative diving patterns of pinnipeds and seabirds. Ecol Monogr 71:137-162

Suzuki I, Naito Y, Folkow L, Miyazaki N, Blix AS (2009) Validation of a device for accurate timing of feeding events in marine animals. Polar Biol 32:667-671 
Weimerskirch H, Doncaster CP, Cuenot-Chaillet F (1994) Pelagic seabirds and the marine environment: foraging patterns of wandering albatrosses in relation to prey availability and distribution. Proc R Soc (Lond) B Biol Sci 255: 91-97

Williams TM, Fuiman LA, Horning M, Davis RW (2004) The cost of foraging by a marine predator, the Weddell seal Leptonychotes weddellii: pricing by the stroke. J Exp Biol 207:973-982

Wilson RP, Cooper J, Plötz J (1992) Short communication: Can we determine when marine endotherms feed? A case study with seabirds. J Exp Biol 167:267-275

Editorial responsibility: Rory Wilson,

Swansea, UK
Wilson RP, Puetz K, Bost CA, Culik BM, Bannasch R, Reins T, Adelung D (1993) Diel dive depth in penguins in relation to diel vertical migration of prey: whose dinner by candlelight? Mar Ecol Prog Ser 94:101-104

> Wilson R, Steinfurth A, Ropert-Coudert Y, Kato A, Kurita M (2002) Lip-reading in remote subjects: an attempt to quantify and separate ingestion, breathing and vocalisation in free-living animals using penguins as a model. Mar Biol 140:17-27

Yoda K, Naito Y, Sato K, Takahashi A and others (2001) A new technique for monitoring the behaviour of free-ranging Adélie penguins. J Exp Biol 204:685-690

Submitted: May 1, 2009; Accepted: September 4, 2009 Proofs received from author(s): December 7, 2009 